\title{
Intracellular Thiol Redox Status Regulates Lymphangiogenesis and Dictates Corneal Limbal Graft Survival
}

\section{Citation}

Fukumoto, Akiko, Kazuichi Maruyama, Tony Walsh, Kentaro Kajiya, Junji Hamuro, Patricia A. D'Amore, and Shigeru Kinoshita. 2010. "Intracellular Thiol Redox Status Regulates Lymphangiogenesis and Dictates Corneal Limbal Graft Survival." Investigative Opthalmology \& Visual Science 51 (5) (May 1): 2450. doi:10.1167/iovs.09-4618.

\section{Published Version}

10.1167/iovs.09-4618

\section{Permanent link}

http://nrs.harvard.edu/urn-3:HUL.InstRepos:35013973

\section{Terms of Use}

This article was downloaded from Harvard University's DASH repository, and is made available under the terms and conditions applicable to Other Posted Material, as set forth at http:// nrs.harvard.edu/urn-3:HUL.InstRepos:dash.current.terms-of-use\#LAA

\section{Share Your Story}

The Harvard community has made this article openly available.

Please share how this access benefits you. Submit a story.

Accessibility 


\title{
Intracellular Thiol Redox Status Regulates Lymphangiogenesis and Dictates Corneal Limbal Graft Survival
}

\author{
Akiko Fukumoto, ${ }^{1}$ Kazuichi Maruyama, ${ }^{1}$ Tony Walsh,${ }^{2}$ Kentaro Kajiya, ${ }^{4}$ Junji Hamuro, ${ }^{1}$ \\ Patricia A. D'Amore, ${ }^{2,3}$ and Shigeru Kinoshita ${ }^{1}$
}

Purpose. Compounds regulating intracellular thiol redox status, such as $N, N$-diacetyl-L-cystine dimethylester $\left(\mathrm{NM}_{2}\right)$, were shown to prolong corneal graft survival in a penetrating keratoplasty (PKP) model. However, the effect of $\mathrm{NM}_{2}$ on hemangiogenesis and lymphangiogenesis has not been investigated. The effect of manipulating ambient thiol redox status on riskier (higher rejection rate) transplantation models, such as limbal graft survival and hemangiogenesis and lymphangiogenesis in a corneal suture model, were investigated.

Methods. C57BL/10 mice that received BALB/c corneas were treated by subconjunctival injection of $\mathrm{NM}_{2}$, and limbal graft survival was assessed. Sutured $\mathrm{C} 57 \mathrm{BL} / 6$ received daily intraperitoneal injections of $\mathrm{NM}_{2}$, glutathione diethylester (GSHOEt), or PBS. Lymphatic endothelial cell (LEC) and peritoneal mps were treated with $\mathrm{NM}_{2}$ or GSHOEt, and then VEGFR3, neuropilin-2, podoplanin, and LYVE-1 expression were analyzed. Supernatants were collected for analysis of TNF- $\alpha$ and VEGF-A levels by ELISA.

RESULTs. Significantly less cellular infiltration was detected in mice with corneal limbal transplant-treated $\mathrm{NM}_{2}$-treated mice. Hemangiogenesis and lymphangiogenesis were suppressed in the $\mathrm{NM}_{2}$-treated mice. $\mathrm{NM}_{2}$ treatment of mps led to reduced levels of VEGFR3, neuropilin-2, podoplanin, and LYVE-1 expression compared with PBS- or GSHOEt- treated mps, lower levels of TNF- $\alpha$, and increased secretion of VEGF. Moreover, $\mathrm{NM}_{2}$-treated LECs had reduced levels of $L Y V E-1$ and Prox-1.

Conclusions. Reduction of ambient redox status reduced inflammatory cell infiltrates. Consequently, reduced inflammatory response might have contributed to both the observed prolonged corneal limbal graft survival and the attenuated hemangiogenesis and lymphangiogenesis in cornea. (Invest Ophthalmol Vis Sci. 2010;51:2450-2458) DOI:10.1167/iovs.09-4618

$\mathrm{L}$ ymphangiogenesis is a critical milestone in tumor progression because the extravasation of tumor cells through lymphatic vessels is thought to play a role in tumor dissemina-

From the ${ }^{1}$ Department of Ophthalmology, Kyoto Prefectural University of Medicine, Kyoto, Japan; the Departments of ${ }^{2}$ Ophthalmology and ${ }^{3}$ Pathology, Harvard Medical School, Schepens Eye Research Institute, Boston, Massachusetts; and ${ }^{4}$ Shiseido Innovative Science Research and Development Center, Yokohama, Japan.

Supported by Grant-in-Aid for Scientific Research 19791283.

Submitted for publication September 9, 2009; revised November 4, 2009; accepted November 30, 2009

Disclosure: A. Fukumoto, None; K. Maruyama, None; T. Walsh, None; K. Kajiya, None; J. Hamuro, None; P.A. D'Amore, None; S. Kinoshita, None

Corresponding author: Kazuichi Maruyama, Department of Ophthalmology, Kyoto Prefectural University of Medicine, 465 Kajii-cho Kamigyo-ku, Kyoto, Japan 602-0841; kmaruyam@koto.kpu-m.ac.jp. tion. ${ }^{1,2}$ The lymphatic system also plays an essential role in the immune response to infectious agents. ${ }^{3}$ Afferent lymphatic vessels are the route through which antigen-presenting cells (APCs) migrate to the regional lymph nodes and lymphoid organs.

The cornea is normally devoid of blood and lymphatic vessels; however, under inflammatory conditions, such as suture placement or corneal transplantation, lymphatic vessels are induced in the corneal stroma. We have previously suggested that lymphangiogenesis correlates with corneal rejection reaction after transplantation. ${ }^{4-6}$ We and others have suggested that inflammatory cells, including mps, support lymphangiogenesis ${ }^{7,8}$ either by transdifferentiating into lymphatic endothelial cells (LECs) and directly incorporating into the lymphatics or by stimulating the division of local LECs. ${ }^{\text {? }}$

With inflammation, such as that induced by lipopolysaccharide, VEGF-C and VEGFR3 expression is induced on peritoneal $\mathrm{CD}_{11 \mathrm{~b}}{ }^{+}$mps. ${ }^{7}$ Newly recruited bone marrow-derived cells or mesenteric cells, such as mps, ${ }^{10}$ are known to produce angiogenic factors such as VEGF-A, -C, -D and TNF- $\alpha$, thereby playing a major role in inducing lymphangiogenesis and angiogenesis in the cornea. ${ }^{10,11}$

$\mathrm{NM}_{2}$ treatment in corneal transplantation models (penetrating keratoplasty $[\mathrm{PKP}]$ ) successfully prolonged graft surviv$\mathrm{al}^{12,13}$ at least in part by reducing the mps production of proinflammatory mediators such as IL-12 and nitric oxide (NO) and by inhibiting the ongoing Th1 immune responses. Tissue stromal inflammatory reactions indirectly influence the cytokine milieu at the sties of inflamed tissue. Glutathione (GSH) constitutes the first line of the cellular defense against oxidative injury. ${ }^{14}$ Intracellular GSH (icGSH) in mps is central to determining the production of IL-12 by regulating MAPK p38 and JNK activities, ${ }^{13-17}$ indicating that icGSH is critical for determining whether the Th1 or Th2 response predominates. ${ }^{15,18}$ Exposure of mps to IFN- $\gamma$ increased the icGSH/ oxidized icGSH (icGSSG) ratio, whereas exposure to IL-4 decreased it. ${ }^{13,17}$ Mps with decreased icGSH are known as oxidative mps (OMps), and those with increased amounts as referred to reductive mps (RMps). ${ }^{16-20}$ The former was pharmacologically induced by $\mathrm{NM}_{2}$, whereas the latter was pharmacologically induced by GSHOEt. ${ }^{13,14,16,19,20} \mathrm{RMps} / \mathrm{Th} 1$ contributes to tissue damage, followed by inflammatory stromal reactions. Then OMps, induced under impaired blood flow, may mediate tissue regeneration. ${ }^{17,19-21}$ The roles of RMps and OMps in the induction of lymphangiogenesis and hemangiogenesis has not been investigated, though the correlation between ambient hypoxia and angiogenesis has been widely documented 22,23

Corneal limbal transplantation (LT) is performed to reconstitute the ocular surfaces of patients lacking corneal epithelial stem cells because of factors such as chemical burns and Stevens-Johnson syndrome. ${ }^{24-26}$ Corneal epithelium stem cells 
are located in the sclerocorneal limbus and function as a source of healthy corneal epithelial cells. ${ }^{4}$ In patients with unilateral and bilateral ocular surface damage, limbal autotransplantation and allogeneic LT, respectively, provide a means for supplying healthy corneal epithelial stem cells. ${ }^{27-30}$ The donor and host limbus and the subepithelial and stromal layers contain a large number of APCs (e.g., dendritic cells and mps) ${ }^{31,32}$ that are responsible for triggering active immune and inflammatory responses. ${ }^{33-36}$ Consequently, LT might be confronted with the efficient host recognition of the donor graft, followed by the acute allosensitization and swift rejection. In addition, the corneal epithelial layer participates in defense against the spontaneous invasion of blood and lymphatic vessels, ${ }^{37,38}$ and abundant lymphatic and blood vessels in the host limbus may participate in maintaining the inflammatory response.

Our previous paper showed that the Th2-biased immune system could block the rejection reaction in the PKP model. ${ }^{12,13}$ Considering the less immune privileged status in limbus of cornea, it is easy to imagine that the LT is far more susceptible to immunologic rejection. In this study, we used $\mathrm{NM}_{2}$ treatment to alter intracellular thiol redox status and tested its effects on cellular infiltration into cornea after LT and whether it can influence graft survival. The effect of changing thiol redox status in mps using $\mathrm{NM}_{2}$ treatment both on corneal hemangiogenesis and on lymphangiogenesis after suture placement was investigated.

\section{Materials ANd Methods}

\section{Animals}

Eight-week-old male C57BL/6 mice purchased from Japan CREA (Shizuoka, Japan) were used in the suture placement model. Seven- to 10-week-old male BALB/C and B10.D2 (H-2 $\left.{ }^{\mathrm{d}}\right)$ mice were used for the limbal transplantation model. All animals were treated in accordance with the ARVO Statement for the Use of Animals in Ophthalmic and Vision Research, and all experiments were approved by the Committee for Animal Research of Kyoto Prefectural University of Medicine.

\section{Modulation of Ambient Redox Status}

$\mathrm{NM}_{2}$ (Bachem, Bubendorf, Switzerland: E-1770) was used to induce oxidation. Glutathione diethylester (GSH-OEt; Sigma, St. Louis, MO) was used to stimulate the reductive conditions. Before limbal transplantation, BALB/c and B10.D2 mice were injected subconjunctivally with $20 \mu \mathrm{g}$ NM2 or GSH-OEt $(50 \mu \mathrm{L})$ using a 30-gauge needle; the controls received saline containing hyaluronic acid. Hosts received three pretransplantation injections with either $\mathrm{NM}_{2}$ or saline (days -7 , $-3,0$ ); donors were treated twice (days $-7,-3$ ). Alternatively, transplant recipients received three intraperitoneal injections of $200 \mu \mathrm{g}$ $(500 \mu \mathrm{L})$; controls were injected with saline only. Transplant donors were injected intraperitoneally twice. Mice with suture placement received $\mathrm{NM}_{2}$ injections of $200 \mu \mathrm{g}(500 \mu \mathrm{L})$ and GSH-OEt $(2 \mathrm{mg} / 100$ $\mu \mathrm{L}$ ) intraperitoneally every day after suture placement for 7 days.

\section{Mouse Limbal Transplantation and Suture Placement in Corneal Stroma}

Before limbal transplantation, all animals were deeply anesthetized with an intraperitoneal injection of 3 to $4 \mathrm{mg}$ ketamine and $0.01 \mathrm{mg}$ xylazine. Briefly, the cornea without scleral tissue was excised along the limbus of the donor eye, and the corneal endothelial cell layer was completely peeled off using two forceps. The corneal epithelium of deeply anesthetized BALB/c hosts was scraped off completely, the adjacent limbal and conjunctival tissues were dissected circumferentially, and the donor corneal tissue with the limbus was placed on the host cornea. Each corneal lenticule was secured around the recipient corneal limbus with eight interrupted 11-0 nylon sutures (MANI, Inc., Tochigi, Japan). Subsequently, a $2 \times 2$-mm hole was placed in the

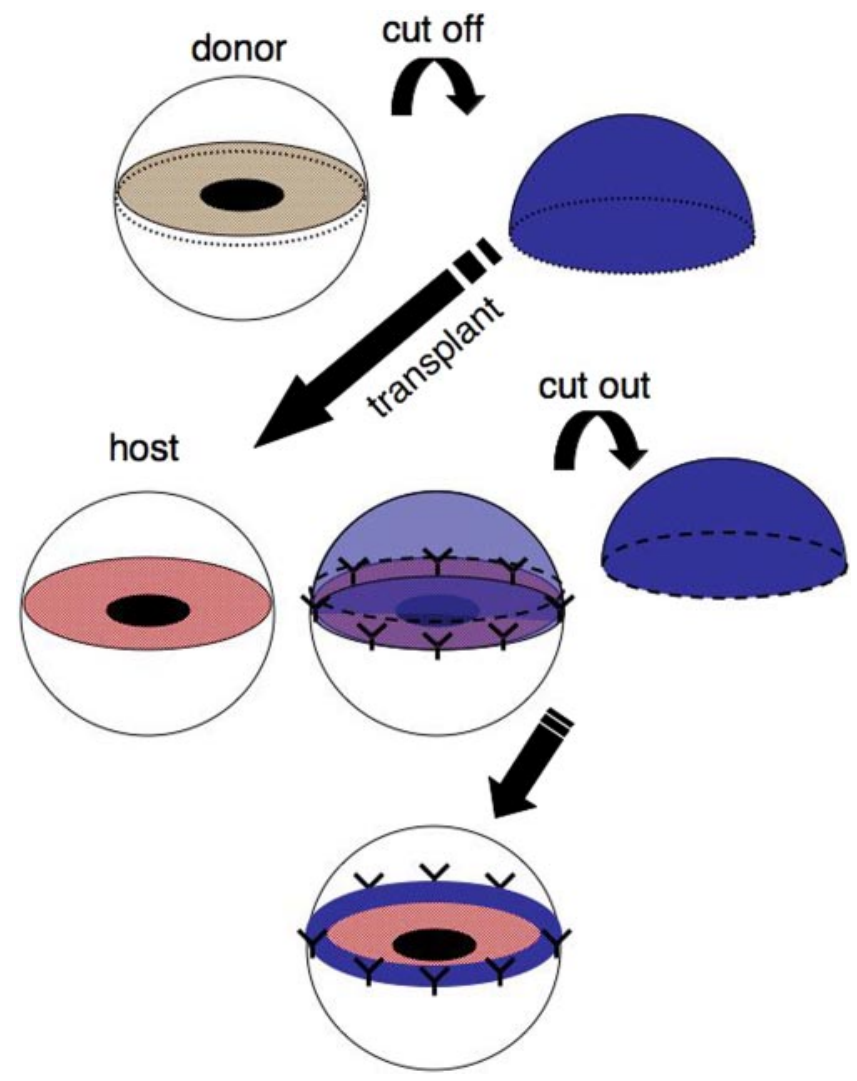

Figure 1. Limbal transplantation model. Cornea, without scleral tissue, was excised along the limbus of the donor eye. The donor corneal endothelial cell layer was then peeled off, and both adjacent limbal and conjunctival tissues were dissected circumferentially. Whole corneal tissue with limbus was grafted onto the host cornea and was secured to the recipient corneal limbus with 8-interrupted 11-0 nylon sutures.

center of the donor cornea, and a cut was made parallel with the host limbus (Fig. 1). Finally, antibiotic ointment was applied to the corneal surface, and the eyelids were sealed for 72 hours with 8-0 nylon tarsorrhaphy (Alcon Surgical, Dallas, TX). Cases that were technically difficult (limbal perforation, anterior chamber hemorrhage during or after surgery, suture loosening, lenticule separation, cataract during the observation period) were excluded from further study. Using stromal incisions that encompassed $>120^{\circ}$ of the corneal circumference, three 11-0 nylon sutures were placed intrastromally. To obtain a standardized angiogenic response, the outer edge of the suture was placed halfway between the limbus and the line outlined by the $2-\mathrm{mm}$ trephine; the inner edge was equidistant from the 2-mm trephine. Sutures were left in place for 7 days.

\section{Evaluation and Scoring of Limbal Transplantation}

After 72 hours the eyelids were opened, and the grafts were evaluated daily under a slit lamp biomicroscope. Corneal reepithelialization was evaluated at 4,7 , and 14 days using $0.25 \%$ methylene blue staining. At each time point, the grafts were scored for opacification using the method previously described, ${ }^{26}$ where 0 represents clear cornea; 1 represents lenticular and regional corneal epithelial edema, opacity, or clearly visible iris vessels; 2 represents diffuse epithelial edema, corneal opacity, or both, and obscuring of the iris vessels; 3 represents diffuse epithelial edema, corneal opacity, or both, and absence of iris vessel visualization; and 4 represents invisibility of the anterior chamber because of epithelial edema, corneal opacity, or both. Regardless of their opacity score at 4 weeks, grafts with opacity scores of 2 or higher were considered rejected (immunologic failure) because some grafts were only transiently opacified. Some mice with an opacification grade 
control
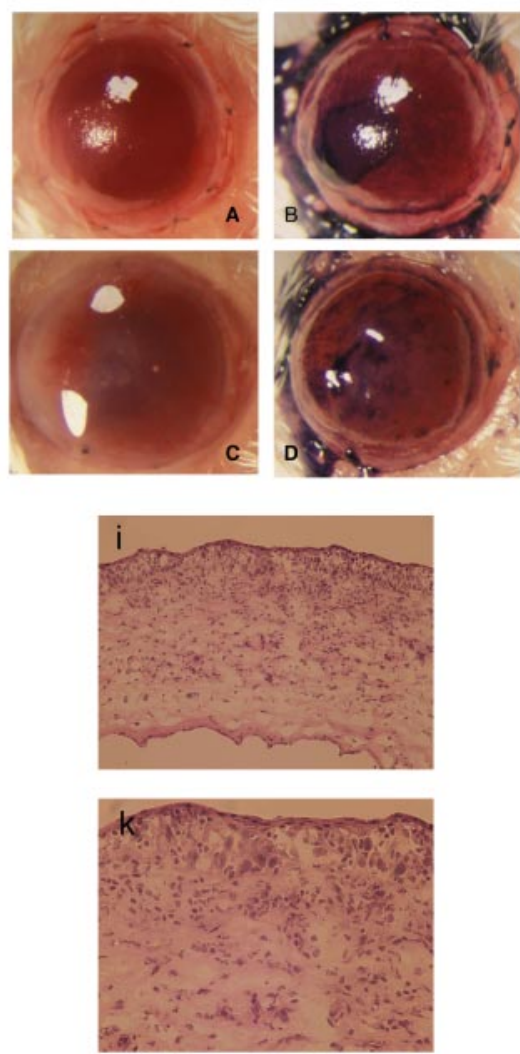

m

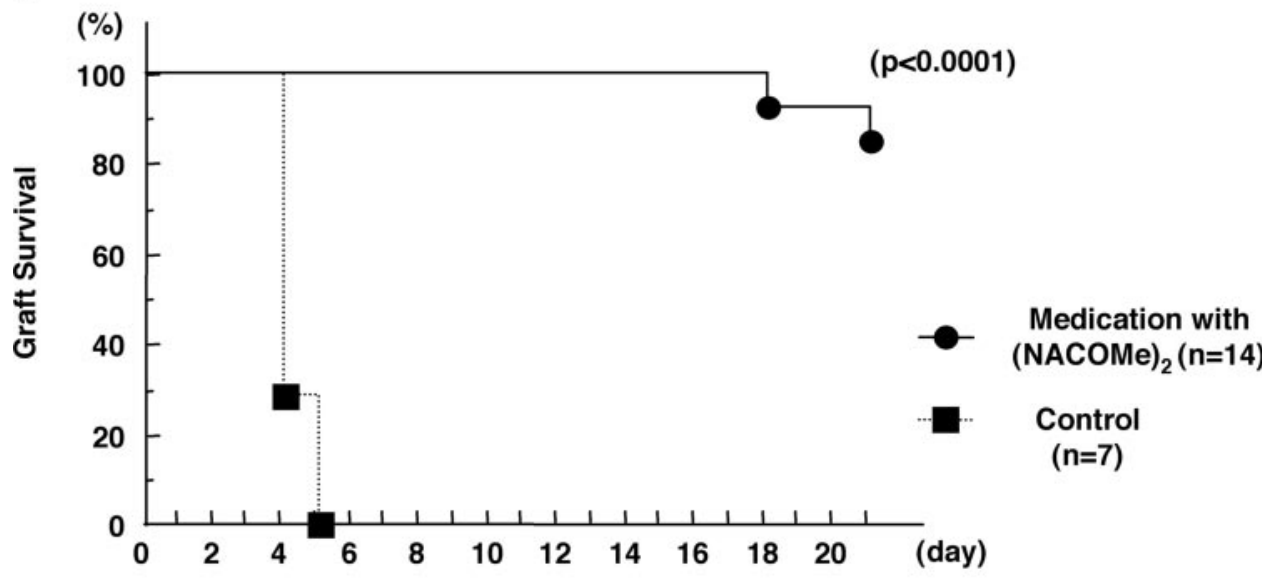

Days after LT
(NACOMe)2
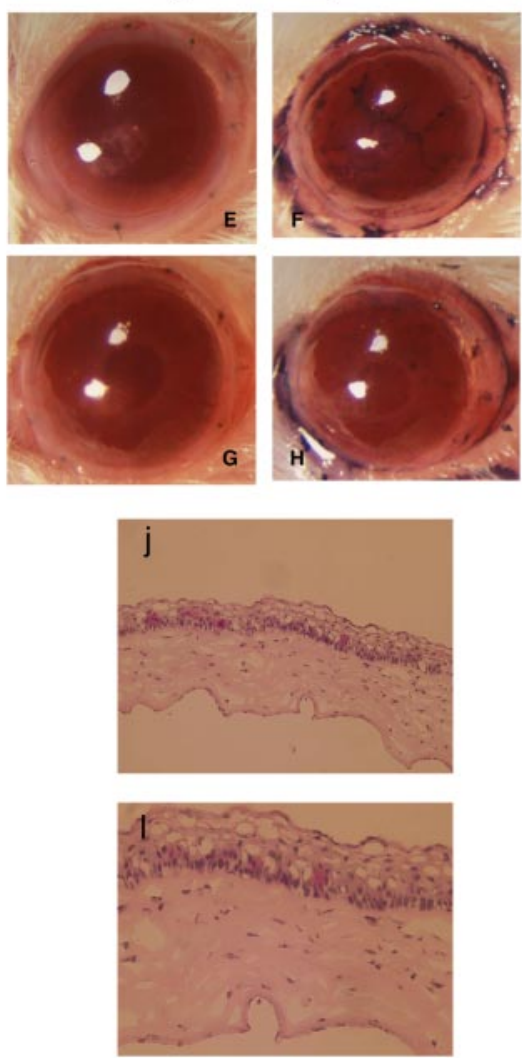

(day)
FiguRE 2. Effect of NM2 treatment on histology and survival of corneal transplants. (a) Control mice typically reject corneas at day 7 . (b) At day 7 , the epithelia of the control group appear defective. (c, d) By day 14 , the corneas of the controls became opacified and exhibited a rough surface. (e, f) In contrast, the corneas of $\mathrm{NM}_{2}$-treated mice were clear on day $7 .(\mathbf{g}, \mathbf{h})$ At day 14 , the $\mathrm{NM}_{2}$-treated corneas remained clear and displayed smooth surfaces. (k) Methylene blue stain revealed reepithelialization. (i, k) The epithelia of the donor lenticule of untreated mice contained many infiltrating cells. ( $\mathbf{j}$, 1) In contrast, the epithelia of $\mathrm{NM}_{2}-$ treated mice showed few invading lymphocytes, and the epithelial cells were aligned in central areas of the cornea. (m) Survival rate after transplantation with or without $\mathrm{NM}_{2} \mathrm{NM}_{2}$ treatment promoted long-term graft survival of minor $\mathrm{H}$ disparate donor allograft. B10.D2 corneal allografts were grafted after treatment of $\mathrm{NM}_{2}$ ( $n=140$ ), or control $(n=7 \mathbf{\square})$. Results are presented as KaplanMeier survival curves. of 2 were euthanatized by cervical dislocation, and their enucleated eyes were examined histologically. The eyes of mice scored as grade 2 contained numerous infiltrating cells with prominent central edema and disarrayed basal corneal epithelium.

\section{Determination of Hemangiogenesis and Lymphangiogenesis}

Mice were euthanatized on day 7 after transplantation. The corneas were excised, rinsed three times in PBS, and fixed in acetone for 1 hour. They were then rinsed once again in PBS, blocked with $2 \%$ BSA-PBS, and incubated overnight at $4{ }^{\circ} \mathrm{C}$ with rabbit anti-mouse LYVE-1 antibody (1:200; Reliatech, Braunschweig, Germany). ${ }^{39,40}$ The tissues were then washed, blocked, and stained overnight with rat anti-mouse CD31 (PECAM-1; 1:100; BD PharMingen), then finally washed, blocked, and stained with FITC- and Cy3-conjugated secondary antibody (1:100; Jackson ImmunoResearch Laboratories, West Grove, PA). Double-stained whole-mount sections were analyzed under a fluorescence microscope (Olympus, Tokyo, Japan) and an inverted and upright confocal laser-scanning microscope (TSC-SP2; Leica, Wetzlar, Germany). Digital pictures of the flat-mounts were taken with an image analysis system (Spot, Chantilly, VA), and the area covered by blood and lymphatic vessels positive for CD31/LYVE-1 ${ }^{39,40}$ was measured using ImageJ software (developed by Wayne Rasband, National Institutes of Health, Bethesda, MD; available at http://rsb. info.nih.gov/ij/index.html). The total corneal area covered by these vessels was outlined using the innermost vessel of the limbal arcade as 

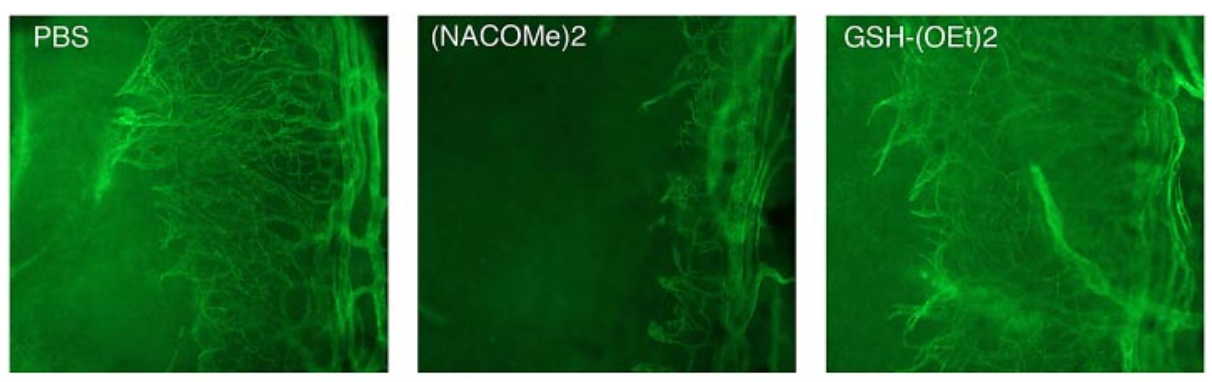

b

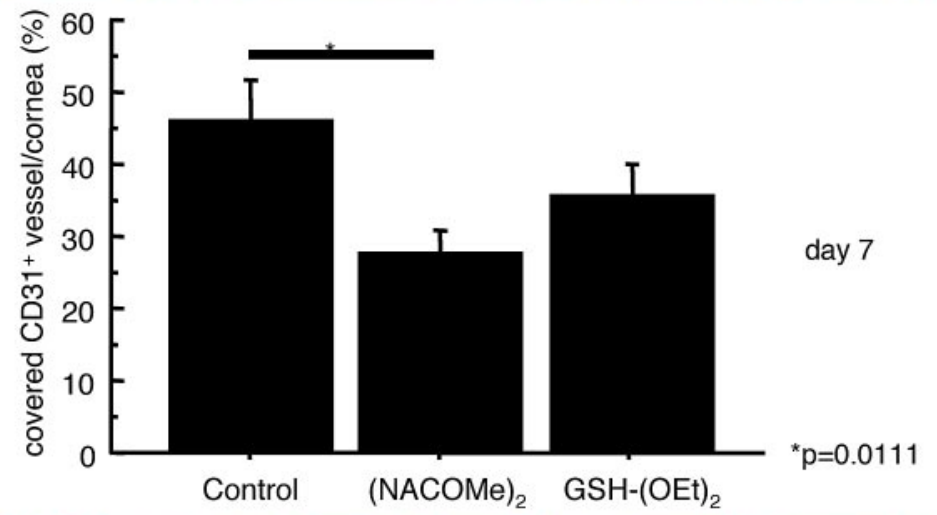

C
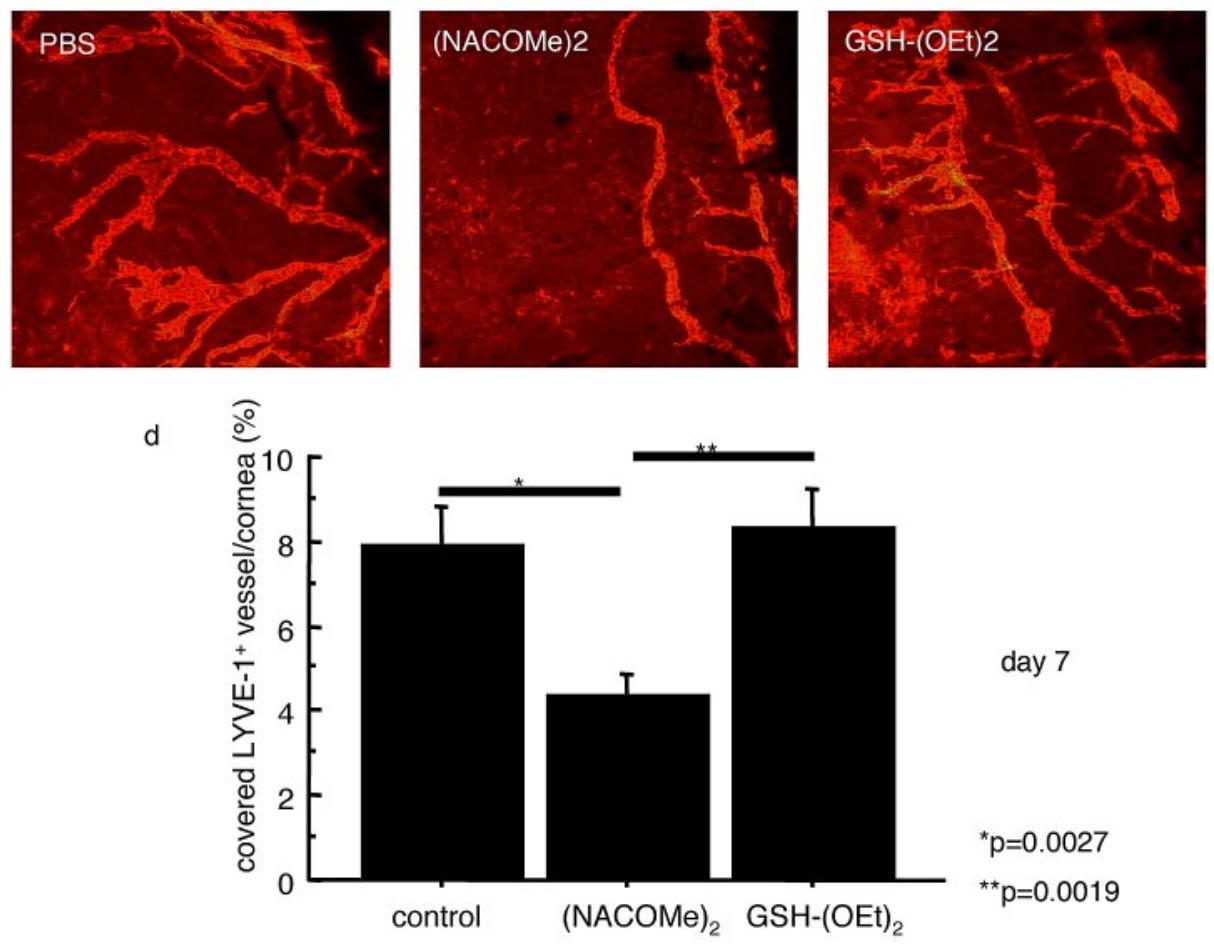

FiguRE 3. Effect of NM2 treatment on hemangiogenesis and lymphangiogenesis after corneal transplantation. (a) Wholemount cornea stained with the pan-endothelial cell marker CD31 (green). (b) Angiogenesis was suppressed in $\mathrm{NM}_{2}$-treated mice compared with PBS-treated mice $\left({ }^{*} P<\right.$ 0.0111). (c) Whole-mount cornea stain with the lymphatic endothelial cell marker LYVE-1 (red). (d) Lymphangiogenesis was significantly suppressed in the $\mathrm{NM}_{2}$-treated mice compared with the GSH-OEt-treated mice $(* * P<0.0019)$ and the PBStreated mice $\left({ }^{*} P<0.0027\right)$. the border; the area of blood and lymphatic neovascularization within the cornea was calculated and normalized to the total corneal area (expressed as a percentage of the cornea covered by vessels).

\section{Collection and Culture of mps from the Peritoneal Cavity}

Thioglycollate-induced mps were collected from the peritoneal cavity (peritoneal exudates cells [PECs]) of healthy 8-week-old male C57BL/6 mice. ${ }^{41}$ PECs were washed, resuspended, and cultured ( 24 hours at $37^{\circ} \mathrm{C}$ in $5 \% \mathrm{CO}_{2}$ and air; $10^{6}$ cell/plate) in $35-\mathrm{mm}$ culture plates in RPMI 1640 medium containing $10 \%$ BSA (Sigma), $1 \times 10^{-5}$ M 2-mercaptoethanol (Sigma), $10 \mathrm{mM}$ HEPES, $0.1 \mathrm{mM}$ nonessential amino acid, $1 \mathrm{mM}$ sodium pyruvate, $100 \mathrm{U} / \mathrm{mL}$ penicillin, and $100 \mu \mathrm{g} / \mathrm{mL}$ streptomycin (BioWhittaker, Walkersville, MD).

\section{RT-PCR, Western Blot Analysis, and ELISA}

For RT-PCR analysis, total RNA was extracted from PECs or LECs (STAT-60; Life Technologies, Rockville, MD). First-strand cDNA was prepared from total RNA by reverse transcriptase using oligo (dT) primers. VEGF-C, VEGFR3, neuropilin (NRP)-2, and VEGFR1 expression were detected with primers by RT-PCR. ${ }^{10}$ Podoplanin protein expression was detected by Western blot analysis using rabbit antimouse podoplanin (kindly gifted by Dontscho Kerjaschki, Vienna, Austria). TNF- $\alpha$ and VEGF-A were measured with ELISA. 


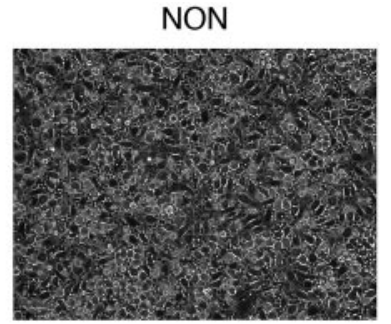

(NACOMe)2

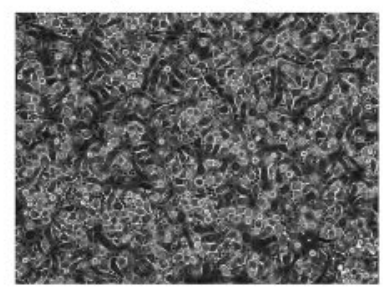

GSH-(OEt)2

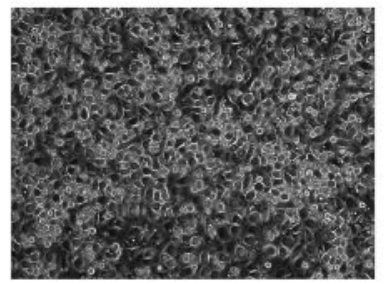

b

NRP-2

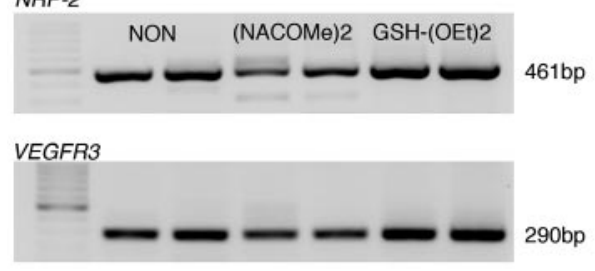

VEGF-C
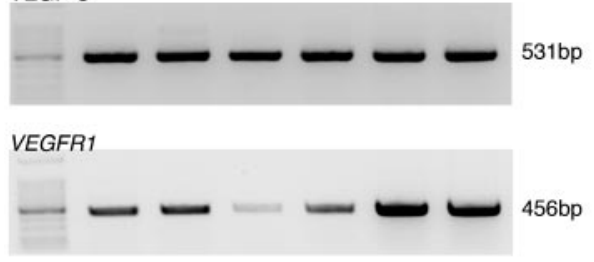

$G A P D H$

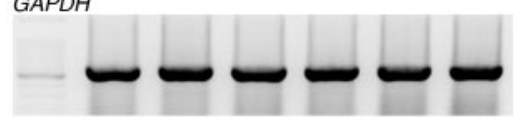

1

d

43kDa
23
C
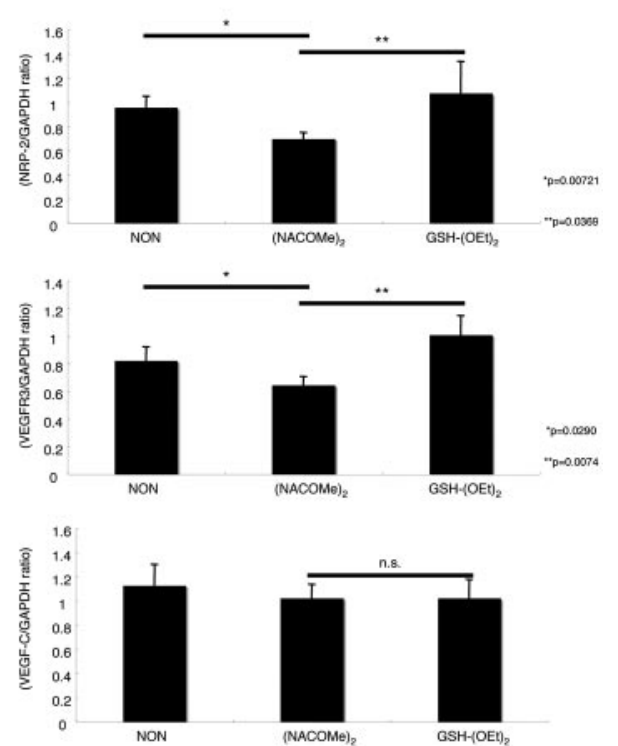

podoplanin

\section{1, Glutathione}

2, NACOMe2

\section{3, Non stimulation}

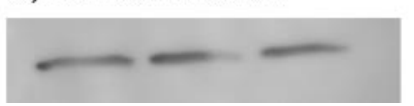

beta actin
FiguRE 4. Effect of $\mathrm{NM}_{2}$ treatment on the PEC morphology and expression of lymphatic-specific markers. (a) Morphology of PEC after $\mathrm{NM}_{2}$ stimulation. (b) RT-PCR of VEGFR3, neuropilin-2, VEGFR1, VEGF-C mRNA expression. (c) Quantification of VEGFR3, neuropilin-2, and VEGF-C expression by RT-PCR. (d) Western blot analysis of podoplanin expression.

\section{Real-Time PCR Analysis}

Total RNA was reverse transcribed (TaqMan Mutiscribe RT Kit; Applied Biosystems, Foster City, CA), and amplification was performed (ABI 7300 Real Time PCR System; Applied Biosystems). The following primers and probes were designed (PrimerExpress software; Applied Biosystems): Prox-1 forward 5'-CTTCCGCCATCCCTTTCC-3', reverse 5'-GGGAGCACCTAGTGGACTCTGA-3', and FAM-TGCCCTTGATGGCTTATCCAT-BHQ; $L Y V E-1$ forward 5'-CAGCAGCAGCGCCTACTTG-3', reverse, 5'-CCGGGTGGTGGCAGAA-3', and FAMTCATCCCCTGACTCCACAACACC-TAMRA.

Relative expression levels of the target gene mRNAs were calculated by the comparative $\mathrm{C}_{\mathrm{T}}$ method. All target sequences were normalized to $18 \mathrm{~S}$ mRNA (VIC-labeled TaqMan reagents; Applied Biosystems) in multiplexed reactions performed in duplicate. Differences in $\mathrm{C}_{\mathrm{T}}$ values were calculated for each target mRNA after subtracting the mean value of $18 \mathrm{~S}$ rRNA (relative expression $=2^{-\mathrm{DCT}}$ ).

\section{Statistical Analysis}

Kaplan-Meier survival curves and the Breslow-Gehan-Wilcoxon test were used to compare allograft survival. The Mann-Whitney $U$ test was used to assess statistical significant for the neovascularization score (NV) score, and Student's $t$-test was used for the proliferation response. $P<0.05$ was considered significant.

\section{RESUlts}

\section{Effect of $\mathrm{NM}_{2}$ on the Fate of the Limbal Transplant}

Each control host $(n=7)$ rejected the corneal limbal graft within 5 days. In contrast, when both donors and recipients ( $n=7$ each) were treated with $\mathrm{NM}_{2}$ before transplantation, the cornea remained clear until the end of the 21-day observation period ( $80 \%$ acceptance, $P<0.001$; Fig. $2 \mathrm{~m}$ ). Reepithelialization of the donor corneal epithelium proceeded from the limbus to the central cornea; the entire corneal surface was reepithelialized within 3 to 4 days after transplantation. Host reepithelialization occurred as reported previously. ${ }^{26}$ In most control mice, the epithelium became progressively opacified, and the corneal surface ceased to be smooth (Figs. 2a-d). In $\mathrm{NM}_{2}$-treated mice, the corneal surface exhibited no opacity on the fifth day after transplantation, and it was completely clear 


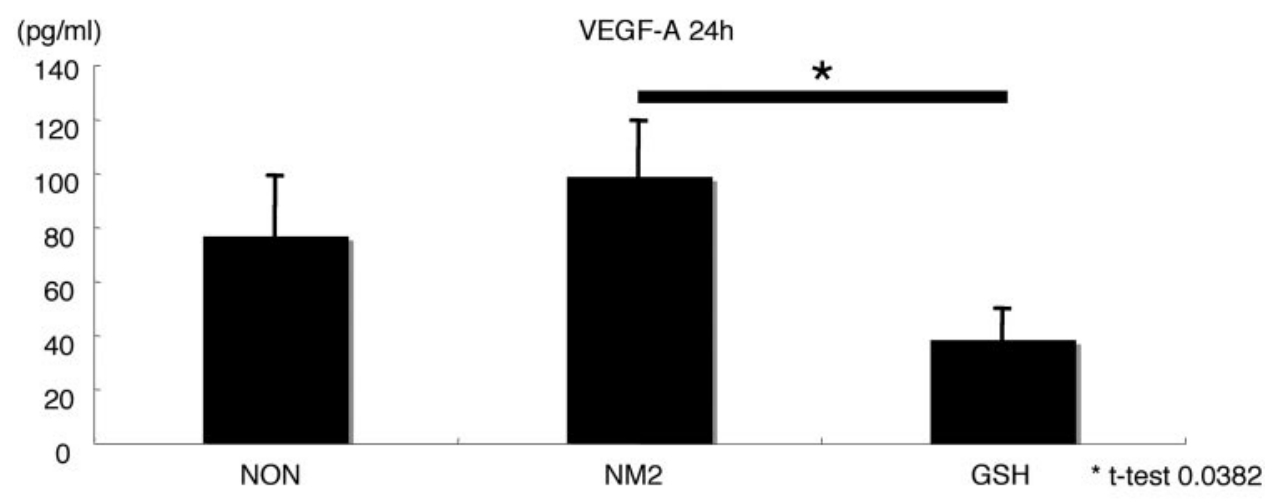

Figure 5. Cytokine secretion by $\mathrm{NM}_{2}$-stimulated PECs. VEGF-A and TNF- $\alpha$ levels, measured by ELISA, in supernatants of PECs 24 hours after stimulation with $\mathrm{NM}_{2}$. (a) VEGF-A secretion from $\mathrm{NM}_{2}$-treated PECs was significantly higher than that from GSH-OEt-pulsed mps ( $P=0.0328)$. Levels of TNF- $\alpha$ released by GSH-OEtpulsed PECs were higher than in $\mathrm{NM}_{2}-$ treated PECs $\left({ }^{*} P=0.0254\right)(b)$.

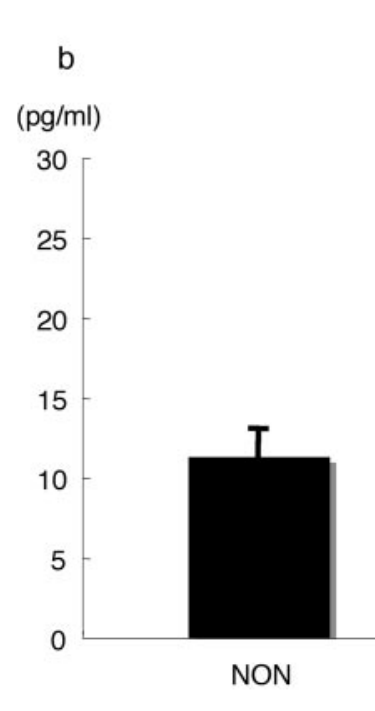

and smooth until day 14 (Figs. $2 \mathrm{e}-\mathrm{h}$ ). Three $\mathrm{NM}_{2}$-treated and control mice were killed on day 7 , and their eyes were examined histologically. The central areas and the subepithelia of the corneas of control mice contained numerous infiltrating cells (Figs. 2i, 2k). In contrast, the epithelium of $\mathrm{NM}_{2}$-treated recipient mice contained few invading inflammatory cells and the epithelium was normally aligned in the central areas of the cornea (Figs. 2j, 21).

\section{Effect of $\mathrm{NM}_{2}$ Treatment on Blood and Lymphatic Vessel Growth in Corneal Suture Model}

At day 7 after suture placement, blood and lymphatic vessel growth in PBS-treated mouse corneas, quantified by the area of CD $31^{+}$, blood, and LYVE- $1^{+}$lymphatic vessels 7 days after suture placement, was equivalent to those of PBS-treated mice. Lymphangiogenesis was significantly suppressed in the $\mathrm{NM}_{2}-$ treated mice compared with GSH-OEt-treated mice $(P<$ $0.0019)$ and PBS-treated control mice $(P<0.0027$; Fig. 3). Angiogenesis was suppressed in $\mathrm{NM}_{2}$-treated mice compared with PBS-treated mice $(P<0.0111$; Fig. 3).

\section{Effect of $\mathrm{NM}_{2}$ on Macrophage Production of Angiogenesis-Related Factors}

$\mathrm{NM}_{2}$-treated PECs became spindle-shaped after 24 hours of culture (Fig. 4a) and led to a reduction in the expression of VEGFR3 (vs. PBS, $P=0.0290$; vs. GSH-OEt, $P=0.0074$ ), neuropilin-2 (vs. PBS, $P=0.0072$; vs. GSH-OEt, $P=0.03,694$ ) (Figs. 4b, 4c). Moreover, VEGFR1 expression was significantly reduced in $\mathrm{NM}_{2}$-treated PECs (Fig. 4b). The downregulation of podoplanin compared with PBS or GSH-OEt-treated PECs was confirmed by Western blot analysis (Fig. 4d). These results suggest that an oxidative milieu leads to reduced expression of lymphatic markers on mps.

The expression of VEGF-C, the ligand for VEGFR3 known to be a stimulator of lymphatic vessel growth, was not changed in $\mathrm{NM}_{2}$-treated peritoneal mps compared with PBS- or GSH-OEttreated mps (Figs. 4b, 4c). The level of VEGF-A released by $\mathrm{NM}_{2}$-treated mps was significantly higher than from GSH-OEttreated mps $(P=0.0328$; Fig. 5a). In contrast, higher levels of TNF- $\alpha$ secretion were detected from GSH-OEt-treated than from $\mathrm{NM}_{2}$-treated mps $(P=0.0254$; Fig. 5b).

\section{Effect of $\mathrm{NM}_{2}$ on the Expression of Lymphatic- Related Factors by LEC}

$\mathrm{NM}_{2}$-treated LECs became polygonal after 24 hours (Fig. 6a) and expressed reduced levels of Prox- and LYVE-1 (Figs. 6b, $6 c$ ), indicating that an ambient oxidative milieu leads to the suppression of lymphatic-related markers on LECs.

\section{Discussion}

Corneal inflammation is associated with corneal limbal transplantation and suture placement. We have shown that corneal 
a

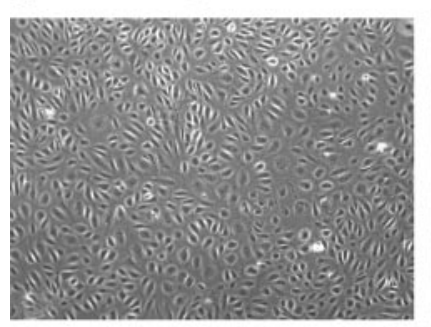

NM2

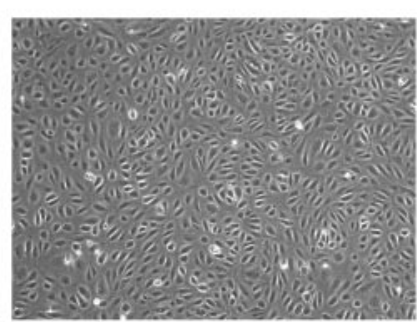

GSHOet

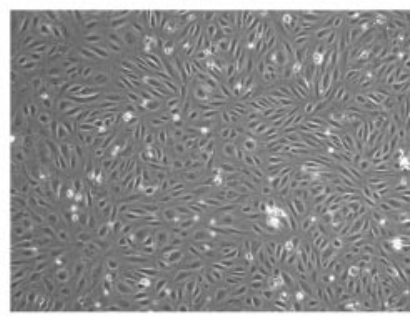

LEC-PROX-1

b

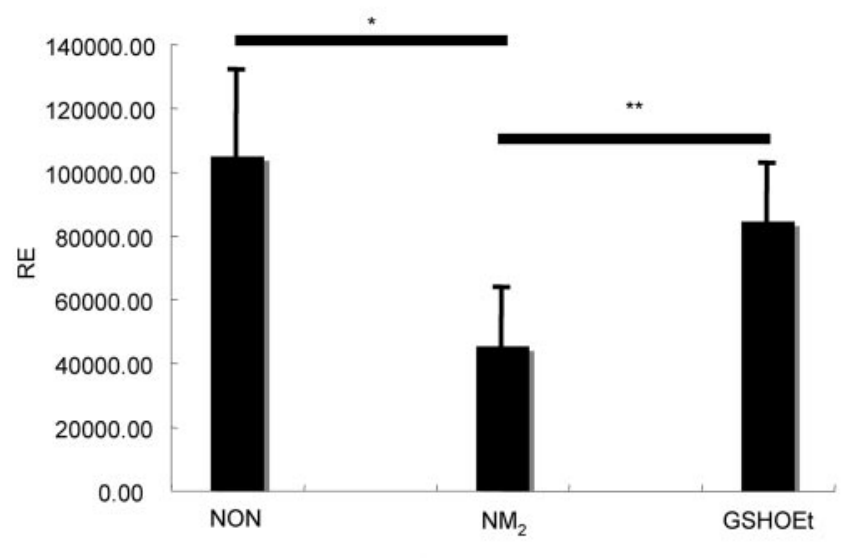

LEC-LYVE-1

C

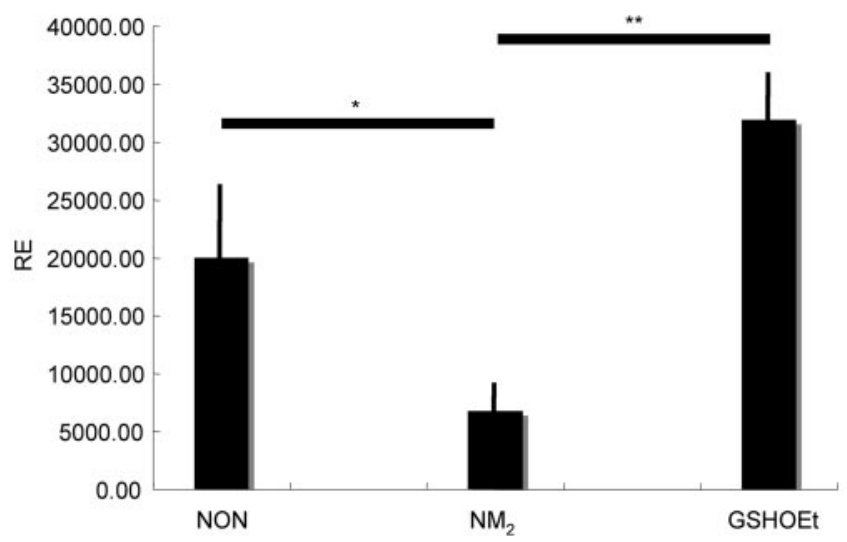

Figure 6. Morphology and the expression of lymphatic- specific markers on LEC. Top: photomicrograph of LEC after $\mathrm{NM}_{2}$ stimulation. Bottom: real-time RT-PCR analysis of Prox-1 $\left({ }^{*} P=0.033,{ }^{* *} P=0.015\right)$ and LYVE-1 ( $\left.P<0.05,{ }^{* *} P=0.015\right)$ expression. inflammation, characterized by cell infiltration, hemangiogenesis, and lymphangiogenesis, were attenuated by pharmacologically skewing ambient thiol redox status to an oxidative status with $\mathrm{NM}_{2}$. The observed in vitro effects of $\mathrm{NM}_{2}$ on mps production of hemangiogenesis- and lymphangiogenesis-related proteins led us to speculate that the ambient redox status resulted in an aggravated inflammatory response that might have contributed to impaired graft survival in patients who underwent corneal limbal transplantation.

Lymphatic and blood vessel growth are both integral in wound healing and tissue remodeling. ${ }^{42-44}$ The role of mps, as a source of diverse mediators and as phagocytes during wound healing, has been well documented. ${ }^{45} \mathrm{Mps}$ recruited to inflamed sites regulates tissue repair and induces the formation of lymphatic and blood vessels. ${ }^{3,4,11}$ We and others ${ }^{10-12}$ have previously shown that monocytes/mps participate in the formation of lymphatic vessels during inflammation. ${ }^{10-12} \mathrm{Mps}$ depletion with clodronate improved graft survival in a PKP model, ${ }^{46}$ demonstrating the central role of mps in pathologic corneal inflammation.
We have previously shown that peritoneal monocytes/mps express markers of lymphatic endothelium, including LYVE-1, podoplanin, and Prox-1, and that mps contribute to the formation of lymphatic vessels during acute inflammation in the cornea. $^{7,8,47}$ We also demonstrated that peritoneal mps form lymphatic vessel-like tubes in vitro in a density-dependent manner. ${ }^{7}$ These observations, together with our current findings, lead us to postulate that the reduction in inflammatory infiltrates in corneal stroma of $\mathrm{NM}_{2}$-treated mice may be the cause of the attenuated lymphatic and blood vessel formation in the suture model.

Mps that reside in the limbal areas of both donor and host corneas may be involved in transplant rejection. In the penetrating keratoplasty model, APCs of recipients, especially mps, played a predominant role in antigen recognition. ${ }^{48} \mathrm{NM}_{2}$ treatment of only donor or recipient corneas did not result in improved graft survival in this LT model; both donor and recipient corneas had to be treated to achieve improved graft survival. We speculate that the rejection reaction depended, at least in part, on the number of APCs in this LT model. This 
hypothesis is supported by our finding that only pretreatment of both donors and recipients with $\mathrm{NM}_{2}$, which induced antiinflammatory APCs, led to suppression of the graft rejection. Previously, we reported that the Th2-biased immune system could suppress not only the rejection reaction but also neovascularization in an LT model. ${ }^{49}$ As discussed, manipulating icGSH by $\mathrm{NM}_{2}$ should have a critical role in determining whether the Th1 or the Th2 response predominates. Inducing the Th2-type response by $\mathrm{NM}_{2}$ treatment would result in less inflammation in this LT model.

The induction of hemangiogenesis and lymphangiogenesis may depend in part on the infiltration of inflamed cells, such as mps, into the corneal stroma after scraping of the epithelium layer or after suture placement. It would be difficult to determine whether hemangiogenesis and lymphangiogenesis could also have been induced by rejection reaction and to what extent that might have contributed to the observed findings. The simple corneal neovascularization model might be more useful for future study. The oxidative condition might have suppressed hemangiogenesis and lymphangiogenesis in this suture placement model.

Our observation of VEGF-A and VEGF-C expression by bone marrow-derived mps in the inflamed corneal stroma ${ }^{10}$ indicate that they are a likely source of these factors during inflammation, when they may function to induce blood and lymphatic vessel formation. ${ }^{50}$ Therefore, we felt it was relevant to try to determine whether $\mathrm{NM}_{2}$-treated mps were functionally distinct from GSH-OE-treated mps, which would have an elevated icGSH. $\mathrm{NM}_{2}$-treated mps exhibited significantly reduced expression of VEGFR3, NRP-2, VEGFR-1, and podoplanin compared with GSH-OE-treated mps. Further, the $\mathrm{NM}_{2}$-treated mps expressed elevated VEGF-A and reduced TNF- $\alpha$ levels in comparison with GSH-OEt-treated mps. We speculate that mps under oxidative conditions (such as induced by IL-4) (13,19-21 $^{\text {or }}$ reductive conditions (such induced by IFN- $\gamma)^{13,14,16,19-21}$ stimulate lymphatic or blood vessel growth by producing angiogenic, lymphangiogenic, or antiangiogenic factors. To date there has been no functional categorization of mps in terms of their effect on lymphangiogenesis. One report ${ }^{51}$ suggests that IFN- $\gamma$-activated mps are not angiogenic, whereas IL- 4 -stimulated mps secrete proangiogenic factors. Despite the elevated secretion of VEGF-A, $\mathrm{NM}_{2}$-treated mps might not have been affected because of their reduced expression of VEGFR-1. Detailed study will be needed to clarify the contrasting effect of $\mathrm{NM}_{2}$, namely the upregulation of VEGF-A and the downregulation of VEGFR-1.

It has been proposed that changes in mps function because of altered icGSH play a role in the pathologic progression of inflammation. It has been reported that $\mathrm{NM}_{2}$ or GSH-OEt administration in mice with autoimmune diabetes results in the inhibition or elevation, respectively, of the Th1-type cytokine milieu. ${ }^{19}$ Our previous studies showed that the suppression of Th1 polarization, followed by skewing to Th2 dominance, prolonged graft survival in a limbal transplantation model. ${ }^{49}$ However, in this model, the cornea grafted to the host with suppressed Th1, skewed Th2 responses elicited few infiltrating cells in contrast to the control cornea, and the recipient did not acquire donor graft-specific tolerance. ${ }^{49}$ The redox status of $\mathrm{mps}$, as measured by icGSH, varies with disease progression in autoimmune mice. ${ }^{19,20}$ At the time of tissue injury, mps skew to reductive mps with elevated icGSH levels, whereas tissue remodeling is associated with oxidative mps with decreased icGSH levels.

The paradigm that the sequential conversion redox status of mps (or other inflammatory cells) dictates the pathologic progression of tissue inflammation and wound healing is consistent with the results observed in this study. We hypothesize that $\mathrm{NM}_{2}^{-}$primed inflammatory cells, including oxidative mps, suppress the Th1-type immune-inflammatory axis. Our data clearly showed that the in vivo administration of $\mathrm{NM}_{2}$ induced oxidative mps, ${ }^{12}$ and histologic analysis revealed a lesser degree of inflammatory cell invasion in the $\mathrm{NM}_{2}$ treatment group than in the controls. These findings point to a therapeutic role for $\mathrm{NM}_{2}$ in the suppression not only of corneal stromal inflammation but of hemangiogenesis and lymphangiogenesis in the cornea.

\section{Acknowledgments}

The authors thank Dontcho Kerjaschki for the kind gift of podoplanin antibody and for many discussions and critical reading of the manuscript.

\section{References}

1. Alitalo K, Carmeliet P. Molecular mechanisms of lymphangiogenesis in health and disease. Cancer Cell. 2002;1:219-227.

2. Alitalo K. Growth factors controlling angiogenesis and lymphangiogenesis. Ugeskr Laeger. 2002;164:3170-3172.

3. Cursiefen C, Cao J, Chen L, et al. Inhibition of hemangiogenesis and lymphangiogenesis after normal-risk corneal transplantation by neutralizing VEGF promotes graft survival. Invest Ophthalmol Vis Sci. 2004;45:2666-2673.

4. Cursiefen C, Chen L, Dana MR, Streilein JW. Corneal lymphangiogenesis: evidence, mechanisms, and implications for corneal transplant immunology. Cornea. 2003;22:273-281.

5. Streilein JW, Yamada J, Dana MR, Ksander BR. Anterior chamberassociated immune deviation, ocular immune privilege, and orthotopic corneal allografts. Transplant Proc. 1999;31:1472-1475.

6. Dana MR, Streilein JW. Loss and restoration of immune privilege in eyes with corneal neovascularization. Invest Ophthalmol Vis Sci. 1996;37:2485-2494.

7. Maruyama K, Ii M, Cursiefen C, et al. Inflammation-induced lymphangiogenesis in the cornea arises from CD11b-positive macrophages. J Clin Invest. 2005;115:2363-2372.

8. Kerjaschki D, Huttary N, Raab I, et al. Lymphatic endothelial progenitor cells contribute to de novo lymphangiogenesis in human renal transplants. Nat Med. 2006;12:230-234.

9. Kerjaschki D. The crucial role of macrophages in lymphangiogenesis. J Clin Invest. 2005;115:2316-2319.

10. Cursiefen C, Chen L, Borges LP, et al. VEGF-A stimulates lymphangiogenesis and hemangiogenesis in inflammatory neovascularization via macrophage recruitment. J Clin Invest. 2004;113: $1040-1050$.

11. Maruyama K, Asai J, Ii M, Thorne T, Losordo DW, D'Amore PA. Decreased macrophage number and activation lead to reduced lymphatic vessel formation and contribute to impaired diabetic wound healing. Am J Patbol. 2007;170:1178-1191.

12. Yamada J, Maruyama K, Sano Y, Kinoshita S, Murata Y, Hamuro J. Promotion of corneal allograft survival by the induction of oxidative macrophages. Invest Ophthalmol Vis Sci. 2004;45:448-454.

13. Yamada J, Hamuro J, Terai K, Kinoshita S. Major histocompatibility complex semi-matching improves murine corneal allograft survival under oxidative macrophage dominancy. Transplantation. 2007; 84:899-907.

14. Meister A. Mechanism and regulation of the glutamine-dependent carbamyl phosphate synthetase of Escherichia coli. Adv Enzymol Relat Areas Mol Biol. 1989;62:315-374.

15. Peterson JD, Herzenberg LA, Vasquez K, Waltenbaugh C. Glutathione levels in antigen-presenting cells modulate Th1 versus Th2 response patterns. Proc Natl Acad Sci US A. 1998;95:3071-3076.

16. Murata Y, Amao M, Yoneda J, Hamuro J. Intracellular thiol redox status of macrophages directs the Th1 skewing in thioredoxin transgenic mice during aging. Mol Immunol. 2002;38:747-757.

17. Murata Y, Yamashita A, Saito T, Sugamura K, Hamuro J. The conversion of redox status of peritoneal macrophages during pathological progression of spontaneous inflammatory bowel disease in Janus family tyrosine kinase $3(-/-)$ and IL-2 receptor gamma(-/-) mice. Int Immunol. 2002;14:627-636. 
18. Murata Y, Shimamura T, Hamuro J. The polarization of T(h)1/T(h)2 balance is dependent on the intracellular thiol redox status of macrophages due to the distinctive cytokine production. Int Immunol. 2002;14:201-212.

19. Murata Y, Amao M, Hamuro J. Sequential conversion of the redox status of macrophages dictates the pathological progression of autoimmune diabetes. Eur J Immunol. 2003;33:1001-1011.

20. Murata Y, Ohteki T, Koyasu S, Hamuro J. IFN-gamma and proinflammatory cytokine production by antigen-presenting cells is dictated by intracellular thiol redox status regulated by oxygen tension. Eur J Immunol. 2002;32:2866-2873.

21. Vayalil PK, Iles KE, Choi J, Yi AK, Postlethwait EM, Liu RM Glutathione suppresses TGF-beta-induced PAI-1 expression by inhibiting p38 and JNK MAPK and the binding of AP-1, SP-1, and Smad to the PAI-1 promoter. Am J Pbysiol Lung Cell Mol Pbysiol. 2007;293:L1281-L1292.

22. Edirisinghe I, Yang SR, Yao H, et al. VEGFR-2 inhibition augments cigarette smoke-induced oxidative stress and inflammatory responses leading to endothelial dysfunction. FASEB J. 2008;22: 2297-2310.

23. Mendoza MG, Robles HV, Romo E, Rios A, Escalante B. Nitric oxide-dependent neovascularization role in the lower extremity disease. Curr Pharm Des. 2007;13:3591-3596.

24. Thoft RA. Keratoepithelioplasty. Am J Opbthalmol. 1984;97:1-6.

25. Turgeon PW, Nauheim RC, Roat MI, Stopak SS, Thoft RA. Indications for keratoepithelioplasty. Arch Ophthalmol. 1990;108:233236.

26. Kinoshita S, Ohashi Y, Ohji M, Manabe R. Long-term results of keratoepithelioplasty in Mooren's ulcer. Ophthalmology. 1991;98: $438-445$

27. Gazitt Y, Callander N, Freytes CO, et al. Peripheral blood stem cell mobilization with cyclophosphamide in combination with G-CSF, GM-CSF, or sequential GM-CSF/G-CSF in non-Hodgkin's lymphoma patients: a randomized prospective study. J Hematother Stem Cell Res. 2000;9:737-748.

28. Akagi K, Ikeda Y, Miyazaki M, et al. Vascular endothelial growth factor-C (VEGF-C) expression in human colorectal cancer tissues. Br J Cancer. 2000;83:887-891.

29. Tsai RJ, Sun TT, Tseng SC. Comparison of limbal and conjunctival autograft transplantation in corneal surface reconstruction in rabbits. Ophthalmology. 1990;97:446-455.

30. Kenyon KR. Limbal autograft transplantation for chemical and thermal burns. Dev Opbthalmol. 1989;18:53-58.

31. Matoba AY, Peeler JS, Niederkorn JY. T cell subsets in the immune rejection of murine heterotopic corneal allografts. Invest Ophthalmol Vis Sci. 1986;27:1244-1254

32. Gillette TE, Chandler JW, Greiner JV. Langerhans cells of the ocular surface. Opbthalmology. 1982;89:700-711.

33. Niederkorn JY, Ross JR, He Y. Effect of donor Langerhans cells on corneal graft rejection. I Invest Dermatol. 1992;99:104S-106S.

34. Teunissen MB. Dynamic nature and function of epidermal Langerhans cells in vivo and in vitro: a review, with emphasis on human Langerhans cells. Histochem J. 1992;24:697-716.
35. Steinman RM. Dendritic cells. Transplantation. 1981;31:151-155.

36. De Jager MP, Linde SP, Nel CJ. [Does a correlation exist between positive wound cultures at the end of an operation and later wound and prosthetic sepsis?]. S Afr J Surg. 1992;30:178-181.

37. Ambati BK, Nozaki M, Singh N, et al. Corneal avascularity is due to soluble VEGF receptor-1. Nature. 2006;443:993-997.

38. Cursiefen C, Chen L, Saint-Geniez M, et al. Nonvascular VEGF receptor 3 expression by corneal epithelium maintains avascularity and vision. Proc Natl Acad Sci US A. 2006;103:11405-11410.

39. Cursiefen C, Schlotzer-Schrehardt U, Kuchle M, et al. Lymphatic vessels in vascularized human corneas: immunohistochemical investigation using LYVE-1 and podoplanin. Invest Ophthalmol Vis Sci. 2002;43:2127-2135.

40. Chang L, Kaipainen A, Folkman J. Lymphangiogenesis new mechanisms. Ann N Y Acad Sci. 2002;979:111-119.

41. Takeuchi M, Alard P, Streilein JW. TGF-beta promotes immune deviation by altering accessory signals of antigen-presenting cells. J Immunol. 1998;160:1589-1597.

42. Jain RK. Molecular regulation of vessel maturation. Nat Med. 2003; 9:685-693.

43. Carmeliet P. Angiogenesis in health and disease. Nat Med. 2003; 9:653-660

44. Folkman J. Angiogenesis in cancer, vascular, rheumatoid and other disease. Nat Med. 1995;1:27-31.

45. Singer AJ, Clark RA. Cutaneous wound healing. $N$ Engl J Med. 1999;341:738-746.

46. Van der Veen G, Broersma L, Dijkstra CD, Van Rooijen N, Van Rij $G$, Van der Gaag R. Prevention of corneal allograft rejection in rats treated with subconjunctival injections of liposomes containing dichloromethylene diphosphonate. Invest Ophthalmol Vis Sci. 1994;35:3505-3515.

47. Schledzewski K, Falkowski M, Moldenhauer G, et al. Lymphatic endothelium-specific hyaluronan receptor LYVE-1 is expressed by stabilin-1(+), F4/80(+), CD11b(+) macrophages in malignant tumours and wound healing tissue in vivo and in bone marrow cultures in vitro: implications for the assessment of lymphangiogenesis. J Pathol. 2006;209:67-77.

48. Slegers TP, Torres PF, Broersma L, van Rooijen N, van Rij G, van der Gaag R. Effect of macrophage depletion on immune effector mechanisms during corneal allograft rejection in rats. Invest $O p b$ thalmol Vis Sci. 2000;41:2239-2247.

49. Maruyama K, Yamada J, Sano Y, Kinoshita S. Th2-biased immune system promotion of allogeneic corneal epithelial cell survival after orthotopic limbal transplantation. Invest Ophthalmol Vis Sci. 2003; $44: 4736$ - 4741

50. Karkkainen MJ, Haiko P, Sainio K, et al. Vascular endothelial growth factor $\mathrm{C}$ is required for sprouting of the first lymphatic vessels from embryonic veins. Nat Immunol. 2004;5:74-80.

51. Kodelja V, Muller C, Tenorio S, Schebesch C, Orfanos CE, Goerdt $\mathrm{S}$. Differences in angiogenic potential of classically vs alternatively activated macrophages. Immunobiology. 1997; 197:478-493. 